Communications in Physics, Vol.23, No. 1 (2013), pp. 21-28

\title{
PRODUCTION CROSS SECTIONS OF AXION IN A STATIC ELECTROMAGNETIC FIELD
}

\author{
DANG VAN SOA \\ Hanoi National University of Education \\ TRAN DINH THAM \\ Pham Van Dong University, Quang Ngai, Vietnam
}

\begin{abstract}
Photon - axion conversions in static electromagnetic fields of the size $a \times b \times c$ are considered in detail by the Feynman diagram methods. The differential cross sections are presented and the numerical evaluations of the total cross section are given. Our result shows that the conversion cross-sections in the electric field are quite small, while in the strong magnetic field, the cross-sections are much enhanced, which can be measurable in current experiments.
\end{abstract}

\section{INTRODUCTION}

In the 1970's, Peccei and H. Quinn [1] have shown that the strong CP problem can be solved by the introduction of a light pseudoscalar particle, called the axion [2]. At present, the axion mass is constrained by laboratory searches [3] and by astrophysical and cosmological considerations [4] to between $10^{-6} \mathrm{eV}$ and $10^{-3} \mathrm{eV}$. If the axion has a mass near the low limit of order $10^{-5} \mathrm{eV}$, it is a good candidate for the dark matter of the universe. Besides that, an axino (the fermionic partner of the axion) naturally appears in SUSY models $[5,6]$, which acquire a mass from three - loop Feynman diagrams in a typical range between a few $\mathrm{eV}$ up to a maximum of $1 \mathrm{keV}[7,8]$. Recently, the candidates for dark matter have been done in extended models of the standard model, such as in the 3-3-1 models [9], or in extra-dimensional gauge fields [10].

A particle, if it has a two - photon vertex, may be created by a photon entering an external electromagnetic (EM) field, the axion is one such particle. So far almost all experiments designed to search for light axions make use of the coupling of the axion to photons. Conversion of the axions into EM power in a resonant cavity was first suggested by Sikivie [11]. He suggested that this method can be used to detect the hypothetical galactic axion flux that would exist if axions were the dark matter of the Universe. Various terrestrial experiments to detect invisible axions by making use of their coupling to photons have been proposed $[12,13]$, and the new results of such experiments appeared recently $[14$, $15]$.

The EM detection of axions in experiments is briefly described as follows: The initial photon of energy $q_{o}$ from the laser (may be better from $\mathrm{X}$ ray) interacts with a virtual photon from the EM field to produce the axion of energy $p_{o}$ and momentum : $p=$ $\sqrt{p_{0}^{2}-m_{a}^{2}}$. The photon beam is then blocked to eliminate everything except the axions, which penetrate the wall because of their extremely weak interaction with ordinary matter( 
such shielding is straightforward for a low-energy laser beam). The axion then interacts with another virtual photon in the second EM field to produce a real photon of energy $q_{o}^{\prime}$, whose detection is the signal for the production of the axion. For details of experimental setups the reader can see Refs. $[12,14]$. In our previous works $[16,17]$, we have calculated the different cross-sections and numerical evaluations for the photon-axion conversions in external EM fields. The purpose of this paper is to calculate total cross sections for the conversion of photons into axions in strong external EM fields, namely static electric and magnetic fields of the flat condensor of the size $a \times b \times c$.

\section{MATRIX ELEMENT}

The axion mass and its couplings to ordinary particles are all inversely proportional to the magnitude $v$ of the vacuum expectation value that spontaneously breaks the $U_{P Q}(1)$ quasisymmetry which was postulated by Peccei and Quinn and of which the axion is the pseudo-Nambu - Goldstone boson. For the axion- photon system a suitable Lagrangian density is given by $[4,11]$

$$
L=-\frac{1}{4} F_{\mu \nu} F^{\mu \nu}+g_{\gamma} \frac{\alpha}{4 \pi} \frac{\phi_{a}}{f_{a}} F_{\mu \nu} \tilde{F}^{\mu \nu}+\frac{1}{2} \partial_{\mu} \phi_{a} \partial^{\mu} \phi_{a}-\frac{1}{2} m_{a}^{2} \phi_{a}^{2}\left[1+O\left(\phi_{a}^{2} / v^{2}\right)\right],
$$

where $\phi_{a}$ is the axion field, $m_{a}$ is its mass, $\tilde{F}_{\mu \nu}=\frac{1}{2} \varepsilon_{\mu \nu \rho \sigma} F^{\rho \sigma}$ and $f_{a}$ is the axion decay constant. It is defined in terms of the axion mass $m_{a}$ by $[11,14]: f_{a}=f_{\pi} m_{\pi} \sqrt{m_{u} m_{d}}\left[m_{a}\left(m_{u}+\right.\right.$ $\left.\left.m_{d}\right)\right]^{-1}$. Interaction of axions to the photons arieses from the triangle loop diagram, in which two vertices are interactions of the photon to electrically charged femion and an another vertex is coupling of the axion with fermion. This coupling is model dependent and is given by: $g_{\gamma}=\frac{1}{2}\left(\frac{N_{e}}{N}-\frac{5}{3}-\frac{m_{d}-m_{u}}{m_{d}+m_{u}}\right)$ where $N=\operatorname{Tr}\left(Q_{P Q} Q_{\text {color }}^{2}\right)$ and $N_{e}=Q_{P Q} Q_{e m}^{2}$. $\operatorname{Tr}$ represents the sume over all left - handed Weyl fermions. $Q_{P Q}, Q_{e m}$, and $Q_{c o l o r}$ are respectively the Peccei- Quinn charge, the electric charge, and one of the generators of $S U(3)_{c}$. In particular in the Dine- Fischler - Srednicki - Zhitnitskii model [19]: $g_{\gamma}($ DFSZ $) \simeq 0.36$, and in the Kim- Shifman- Vainshtein- Zakharov model [20] (where the axions do not couple to light quarks and leptons $): g_{\gamma}(\mathrm{KSVZ}) \simeq-0.97$.

Consider the conversion of the photon $\gamma$ with momentum q into the axion $a$ with momentum $\mathrm{p}$ in an external electromagnetic field. For the above mentioned process, the relevant coupling is the second term in (1). Using the Feynman rules we get the following expression for the matrix element

$$
\langle p|\mathcal{M}| q\rangle=-\frac{g_{a \gamma}}{2(2 \pi)^{2} \sqrt{q_{0} p_{0}}} \varepsilon_{\mu}(\vec{q}, \sigma) \varepsilon^{\mu \nu \alpha \beta} q_{\nu} \int_{V} e^{i \vec{k} \vec{r}} F_{\alpha \beta}^{\text {class }} d \vec{r}
$$

where $\vec{k} \equiv \vec{q}-\vec{p}$ is the momentum transfer to the EM field, $g_{a \gamma} \equiv g_{\gamma} \frac{\alpha}{\pi f_{a}}=g_{\gamma} \alpha m_{a}\left(m_{u}+\right.$ $\left.m_{d}\right)\left(\pi f_{\pi} m_{\pi} \sqrt{m_{u} m_{d}}\right)^{-1}$ and $\varepsilon^{\mu}(\vec{q}, \sigma)$ represents the polarization vector of the photon.

Expression (2) is valid for an arbitrary external EM field. In the following we shall use it for conversions in the electric and magnetic fields of the size $a \times b \times c$. Here we use the following notations: $q \equiv|\vec{q}|, p \equiv|\vec{p}|=\left(p_{o}^{2}-m_{a}^{2}\right)^{1 / 2}$ and $\theta$ is the angle between $\vec{p}$ and $\vec{q}$. 


\section{CONVERSIONS IN THE STATIC ELECTRIC FIELD}

Now we take the EM field as a homogeneous electric field of a flat condensor of size $a \times b \times c$. We shall use the coordinate system with the $\mathrm{x}-$ axis parallel to the direction of the field, i.e., $F^{10}=-F^{01}=E$ then the matrix element is given by

$$
\left\langle p\left|\mathcal{M}^{e}\right| q\right\rangle=\frac{g_{a \gamma}}{(2 \pi)^{2} \sqrt{q_{0} p_{0}}} \varepsilon_{\mu}(\vec{q}, \sigma) \varepsilon^{\mu \nu 01} q_{\nu} F_{e}(\vec{k}),
$$

where a form factor for the electric region

$$
F_{e}(\vec{k})=\int_{V} e^{i \vec{k} \vec{r}} E(\vec{r}) d \vec{r}
$$

The superscript e in $M^{e}$ refers to the process taking place in the presence of an electric field. For a homogeneous electric field of intensity $\mathrm{E}$ we have [16]

$$
F_{e}(\vec{k})=8 E \sin \left(\frac{1}{2} a k_{x}\right) \sin \left(\frac{1}{2} b k_{y}\right) \sin \left(\frac{1}{2} c k_{z}\right)\left(k_{x} k_{y} k_{z}\right)^{-1} .
$$

Substituting (4) into (3) we find finally the differential cross section (DCS) for the conversion

$$
\frac{d \sigma^{e}(\gamma \rightarrow a)}{d \Omega}=\frac{g_{a \gamma}^{2} E^{2}}{2(2 \pi)^{2}}\left[\frac{\sin \left(\frac{1}{2} a k_{x}\right) \sin \left(\frac{1}{2} b k_{y}\right) \sin \left(\frac{1}{2} c k_{z}\right)}{k_{x} k_{y} k_{z}}\right]^{2}\left(q_{y}^{2}+q_{z}^{2}\right)
$$

From (5) we see that if the photon moves in the direction of the electric field i.e., $q^{\mu}=$

$(q, q, 0,0)$ then DCS vanishes. If the momentum of the photon is parallel to the $\mathrm{y}-$ axis, i.e., $q^{\mu}=(q, 0, q, 0)$ then Eq.(5) becomes

$$
\begin{aligned}
\frac{d \sigma^{e}(\gamma \rightarrow a)}{d \Omega^{\prime}} & =\frac{32 g_{a \gamma}^{2} E^{2} q^{2}}{(2 \pi)^{2}}\left[\sin \left(\frac{\operatorname{apsin} \theta \sin \varphi^{\prime}}{2}\right) \sin \left(\frac{b}{2}(q-p \cos \theta)\right)\right. \\
& \left.\times \sin \left(\frac{c p \sin \theta \cos \varphi^{\prime}}{2}\right)\right]^{2}\left(p^{2} \sin ^{2} \theta \sin \varphi^{\prime} \cos \varphi^{\prime}(q-p \cos \theta)\right)^{-2} .
\end{aligned}
$$

where $\varphi^{\prime}$ is the angle between the $\mathrm{z}$ - axis and the projection of $\vec{p}$ on the $\mathrm{xz}$ - plane. From (6) we have

$$
\frac{d \sigma^{e}(\gamma \rightarrow a)}{d \Omega^{\prime}}=\frac{2 g_{a \gamma}^{2} E^{2} a^{2} c^{2}}{(2 \pi)^{2} q^{2}\left(1-\sqrt{1-\frac{m_{a}^{2}}{q^{2}}}\right)^{2}} \sin ^{2}\left[\frac{q b}{2}\left(1-\sqrt{1-\frac{m_{a}^{2}}{q^{2}}}\right)\right]
$$

for $\theta \approx 0$ and

$$
\frac{d \sigma^{e}(\gamma \rightarrow a)}{d \Omega^{\prime}}=\frac{8 g_{a \gamma}^{2} a^{2} E^{2}}{(2 \pi)^{2}\left(q^{2}-m_{a}^{2}\right)} \sin ^{2}\left(\frac{b q}{2}\right) \sin ^{2}\left(\frac{c q}{2} \sqrt{1-\frac{m_{a}^{2}}{q^{2}}}\right)
$$

for $\theta=\frac{\pi}{2}, \varphi^{\prime}=0$.

In the limit $m_{a}^{2} \rightarrow 0$, Eqs. (7) and (8) become, respectively,

$$
\frac{d \sigma^{e}(\gamma \rightarrow a)}{d \Omega^{\prime}}=\frac{g_{a \gamma}^{2} q^{2} V^{2} E^{2}}{2(2 \pi)^{2}}+O\left(m_{a}^{4}\right)
$$


and

$$
\frac{d \sigma^{e}(\gamma \rightarrow a)}{d \Omega^{\prime}}=\frac{8 g_{a \gamma}^{2} a^{2} E^{2}}{(2 \pi)^{2} q^{2}} \sin ^{2}\left(\frac{b q}{2}\right) \sin ^{2}\left(\frac{c q}{2}\right)+O\left(m_{a}^{4}\right)
$$

From (9) we see that DCS in the direction of the axio motion depends quadratically on the intensity $\mathrm{E}$, the volume $\mathrm{V}$ of condensor, and the photon momentum $\mathrm{q}$. This is the best case for the conversion.

To evaluate the total cross-section from the general formula (6) we use the parameters chosen as in Refs. [16, 17], $a=b=c=1 \mathrm{~m}$, the intensity of the electric field $E=\frac{100 \mathrm{kV}}{\mathrm{m}}$ and $m_{a}=10^{-5} \mathrm{eV}$. The total cross-section on the selected range of the provided momenta, $q=0.1 \div 1 \mathrm{eV}$, are given in Fig. 1. The upper plot is depicted as 300 points, and the lower one is for 3000 points. We can see from the figures that the cross-sections are quite small to be measurable because of the current experimental limits.

\section{CONVERSIONS IN THE MAGNETIC FIELD}

Now we take that the EM field is the homogeneous magnetic field of the flat condensor of the size $a \times b \times c$. We shall use the coordinate system with the $\mathrm{z}$ - axis parallel to the direction of the field, i.e., $F^{12}=-F^{21}=B$ then the matrix element is given by

$$
\left\langle p|\mathcal{M}| q \angle=\frac{g_{a \gamma}}{(2 \pi)^{2} \sqrt{q_{0} p_{0}}} \varepsilon_{\mu}(\vec{q}, \sigma) \varepsilon^{\mu \nu 21} q_{\nu} F_{m}(\vec{k}),\right.
$$

where a form factor for the magnetic region

$$
F_{m}(\vec{k})=\int_{V} e^{i \vec{k} \vec{r}} B(\vec{r}) d \vec{r}
$$

For a homogeneous magnetic field of intensity B we have

$$
F_{m}(\vec{k})=8 B \sin \left(\frac{1}{2} a k_{x}\right) \sin \left(\frac{1}{2} b k_{y}\right) \sin \left(\frac{1}{2} c k_{z}\right)\left(k_{x} k_{y} k_{z}\right)^{-1} .
$$

Substituting (12) into (11) we find finally the DCS for the conversion of photons into axions

$$
\frac{d \sigma^{m}(\gamma \rightarrow a)}{d \Omega}=\frac{g_{a \gamma}^{2} q^{2}}{2(2 \pi)^{2}}\left(1-\frac{q_{z}^{2}}{q^{2}}\right) F_{m}^{2}
$$

From (13) we see that if the photon moves in the direction of the magnetic field i.e., $q^{\mu}=(q, 0,0, q)$ then DCS vanishes. If the momentum of the photon is parallel to the $\mathrm{x}$ axis, i.e., $q^{\mu}=(q, q, 0,0)$ then Eq.(13) becomes

$$
\begin{aligned}
\frac{d \sigma^{m}(\gamma \rightarrow a)}{d \Omega^{\prime}}= & \frac{32 g_{a \gamma}^{2} B^{2} q^{2}}{(2 \pi)^{2}}\left[\sin \left(\frac{a}{2}(q-p \cos \theta)\right) \sin \left(\frac{b p \sin \theta \cos \varphi^{\prime}}{2}\right)\right. \\
& \left.\times \sin \left(\frac{c p \sin \theta \sin \varphi^{\prime}}{2}\right)\right]^{2}\left(p^{2} \sin ^{2} \theta \sin \varphi^{\prime} \cos \varphi^{\prime}(q-p \cos \theta)\right)^{-2}
\end{aligned}
$$



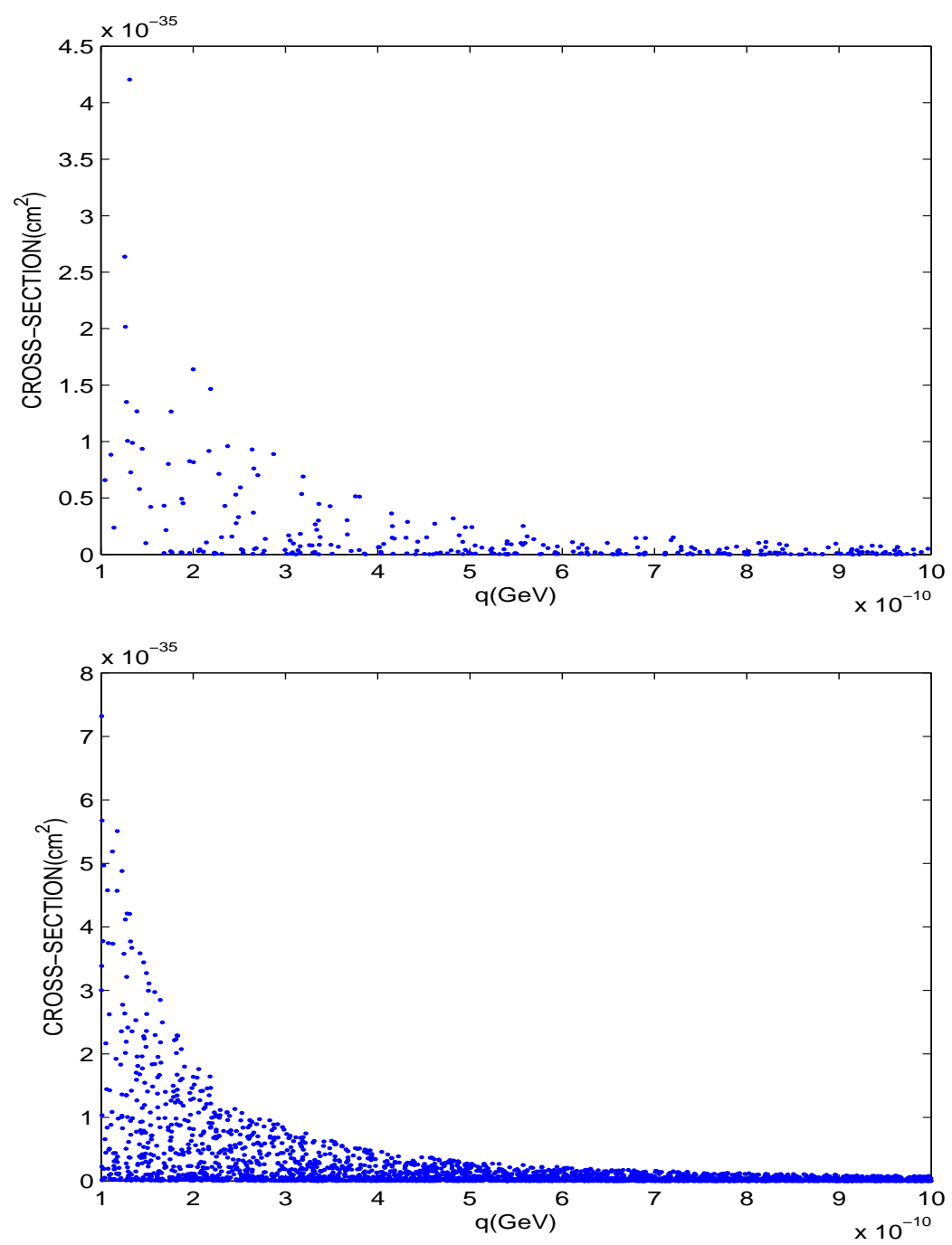

Fig. 1. The total cross-section $\left(\mathrm{cm}^{2}\right)$ for the photon-axion conversion in an electric field as a function of provided momentum $q=0.1 \div 1 \mathrm{eV}$. The upper plot is depicted as 300 points, and the lower one is for 3000 points.

where $\varphi^{\prime}$ is the angle between the y - axis and the projection of $\vec{p}$ on the yz - plane. It is easy to see that

$$
\frac{d \sigma^{m}(\gamma \rightarrow a)}{d \Omega^{\prime}}=\frac{2 g_{a \gamma}^{2} b^{2} c^{2} B^{2}}{(2 \pi)^{2}\left(1-\sqrt{1-\frac{m_{a}^{2}}{q^{2}}}\right)^{2}} \sin ^{2}\left[\frac{q a}{2}\left(1-\sqrt{1-\frac{m_{a}^{2}}{q^{2}}}\right)\right]
$$


for $\theta=0$ and

$$
\frac{d \sigma^{m}(\gamma \rightarrow a)}{d \Omega^{\prime}}=\frac{8 g_{a \gamma}^{2} b^{2} B^{2}}{(2 \pi)^{2}\left(q^{2}-m_{a}^{2}\right)} \sin ^{2}\left(\frac{a q}{2}\right) \sin ^{2}\left(\frac{c q}{2} \sqrt{1-\frac{m_{a}^{2}}{q^{2}}}\right)
$$

for $\theta=\frac{\pi}{2}, \varphi^{\prime}=\frac{\pi}{2}$

$$
\frac{d \sigma^{m}(\gamma \rightarrow a)}{d \Omega^{\prime}}=\frac{8 g_{a \gamma}^{2} c^{2} B^{2}}{(2 \pi)^{2}\left(q^{2}-m_{a}^{2}\right)} \sin ^{2}\left(\frac{a q}{2}\right) \sin ^{2}\left(\frac{b q}{2} \sqrt{1-\frac{m_{a}^{2}}{q^{2}}}\right)
$$

for $\theta=\frac{\pi}{2}, \varphi^{\prime}=0$. For the high energy limit $m_{a} \ll q$, Eqs.(15),(16) and (17) become, respectively,

$$
\begin{gathered}
\frac{d \sigma^{m}(\gamma \rightarrow a)}{d \Omega^{\prime}}=\frac{g_{a \gamma}^{2} q^{2} V^{2} B^{2}}{2(2 \pi)^{2}} \\
\frac{d \sigma^{m}(\gamma \rightarrow a)}{d \Omega^{\prime}}=\frac{8 g_{a \gamma}^{2} b^{2} B^{2}}{(2 \pi)^{2} q^{2}} \sin ^{2}\left(\frac{a q}{2}\right) \sin ^{2}\left(\frac{c q}{2}\right),
\end{gathered}
$$

and

$$
\frac{d \sigma^{m}(\gamma \rightarrow a)}{d \Omega^{\prime}}=\frac{8 g_{a \gamma}^{2} c^{2} B^{2}}{(2 \pi)^{2} q^{2}} \sin ^{2}\left(\frac{a q}{2}\right) \sin ^{2}\left(\frac{b q}{2}\right),
$$

From (18) we see that DCS in the direction of the photon motion depends quadratically on the intensity $B$, the volume $V$ of condensor, and the photon momentum $q$. Therefore, since the external EM field is classical we can increase the scattering probability as much as possible by increasing the intensity of the field or the volume containing the field.

To evaluate the total cross-section from the general formula (14), the parameter values are given as before and $B=9$ Tesla $=9 \times 195.35 \mathrm{eV}^{2}$ The total cross-sections on the selected range of momenta $q$ is presented in Fig. 2. From the figures we see that the total cross-sections for the axion production in the strong magnetic field are much bigger than that of the electric field $\left(\sigma \simeq 10^{-26} \mathrm{~cm}^{2}\right)$, which can be measurable in current experiments.

\section{CONCLUSION}

With the help of the coupling of axion to photons, we have obtained the conversion cross-sections of photon into axion in the presence of several static external fields, including the static electric and strong magnetic fields of the size $a \times b \times c$. The numerical evaluations of the total cross-section are also given. Our result shows that the production crosssections of axion in the static electric field with parameters chosen is quite small, which is not expected to be easily observed. However, the cross-sections in the strong magnetic field are much enhanced, which can be measurable in the current experiments.

Finally, in this work we have considered only a theoretical basis for the experiments, other techniques concerning construction and particle detection can be found in Ref. [21].

\section{ACKNOWLEDGEMENTS}

This research is funded by Vietnam National Foundation for Science and Technology Development (NAFOSTED) under grant number 103.03-2012.80. 

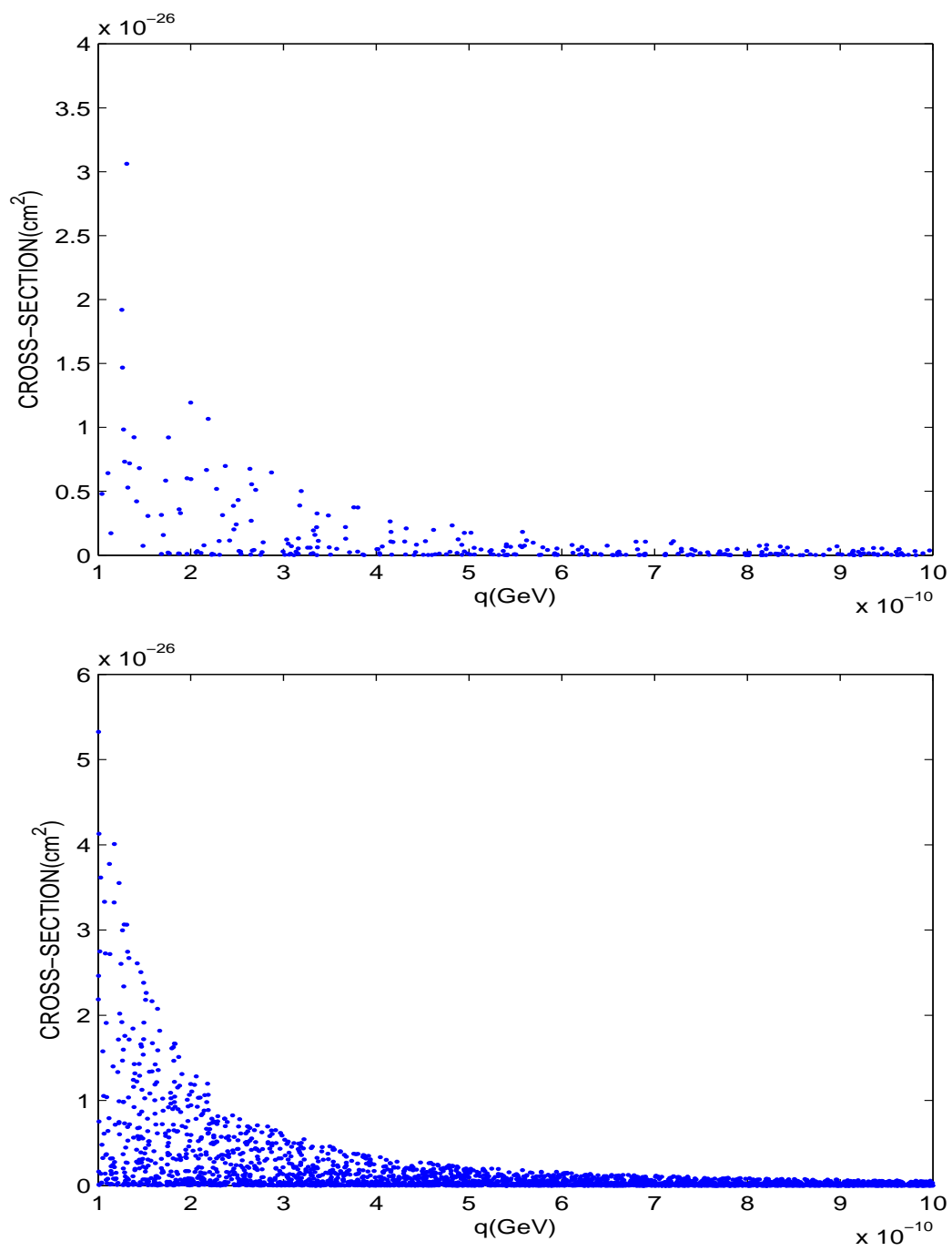

Fig. 2. The total cross-section $\left(\mathrm{cm}^{2}\right)$ for the photon-axion conversion in magnetic field as a function of provided momentum. The upper plot is depicted as 300 points, and the lower one is for 3000 points.

\section{REFERENCES}

[1] R. D. Peccei and H. Quinn, Phys. Rev. Lett. 38 (1977) 1440; Phys. Rev. D16 (1977) 1792.

[2] S. Weinberg, Phys. Rev. Lett. 40 (1977) 223; F. Wilczek, ibid. 40 (1977) 279.

[3] J. E. Kim, Phys. Rep. 150 (1987) 1; H. Y. Cheng, ibid. 158 (1988) 1 ; R. D. Peccei, in CP Violation, edited by C. Jarlskog (Advanced Series on Directions in High Energy Physics, Vol. 3) (World Scientific, Singapore, 1989).

[4] M. S. Turner, Phys. Rep. 197 (1990) 67; G. G. Raffelt, ibid. 198 (1990) 1; E. W. Kolb and M. S. Turner, The Early Universe, Addison- Wesley Publ. Company, 1990.

[5] J. E. Kim, Phys. Lett. B136 (1984) 387; J. E. Kim and H. P. Nilles, ibid.138 (1984) 150. 
[6] K. Rajagopal, M.S. Turner and F. Wilczek, Nucl. Phys. B358 (1991) 447.

[7] M. I. Vysotsky and M. B. Voloshin, Yad. Fiz. 44 (1986) 845; M. B. Voloshin, M. I. Vysotsky and L. B. Okun, Zh. Eksp. Teor. Fiz. 91 (1986) 745 [ Sov. Phys. JETP 64 (1986) 446].

[8] J. E. Kim, A. Masiero and D. V. Nanopoulos, Phys. Lett. B139 (1984) 346.

[9] Alex G. Dias and V. Pleitez, Phys. Rev. D69 (2004) 077702.

[10] Y.Burnier and F.Kuhnel, Phys. Rev. D83 (2011) 115002.

[11] P. Sikivie, Phys. Rev. Lett. 51 (1983) 1415; Phys. Rev. D32 (1985) 2988.

[12] K. Van Bibber, N. R. Dagdeviren, S. E. Koonin, A. K. Kerman, and H. N. Nelson, Phys. Rev. Lett. 59 (1987) 759 ; K. Van Bibber, P. M. McIntyre, D. E. Morris, and G. G. Raffelt, Phys. Rev. D39 (1989) 2089.

[13] S. DePanfilis, A. C. Melissinos, B. E. Moskowitz, J. T. Rogers, Semertzidis, W. U. Wuensch, H. J. Halama, A. G. Prodell, W. B. Fowler, and F. A. Nezrick, Phys. Rev. Lett. 59 (1987) 839.

[14] C. Hagman, P. Sikivie, N. S. Sullivan, and D. B. Tanner, Phys. Rev. D42 (1990) 1297. For details see, Dark Matter in Cosmology Clocks and Test of Fundamental Laws, edited by B. Guiderdoni, G. Greene, D. Hinds, and J. Tran Thanh Van, Editions Frontieres, 1995.

[15] M. Minowa, S. Moriyama, Y. Inoue, T. Namba, Y. Takasu, and A. Yamamoto, Nucl. Phys. B72 (1999) 171.

[16] H. N. Long, D. V. Soa, T. A. Tran, Phys. Lett. B357 (1995) 469.

[17] D. V. Soa, H. N. Long and L. N. Thuc, Mod. Phys. Lett. A22(19) (2007) 1411.

[18] S. Moriyama, M. Minowa, T. Namba, Y. Inoue, Y. Takasu, and A. Yamamoto, Phys. Lett. B434 (1998) 147.

[19] M. Dine, W. Fischler, and M. Srednicki, Phys. Lett. B104 (1981) 199; A. P. Zhitnitskii, Yad. Fiz. 31 (1980) 497 [Sov. J. Nucl. Phys. 31 (1980) 260].

[20] J. E. Kim, Phys. Rev. Lett. 40 (1977) 223; M.A. Shifman, A. I. Vainshtein, and V. I. Zakharov, Nucl. Phys. B166 (1980) 493.

[21] S. Andriamonie et al., The CERN Axion Solar Telescope (CAST): An update, Nucl. Phys. Proc. Suppl. 138 (2005) 41

Received 25 May 2012. 